Proceedings

\title{
High times for cannabis-related videos on YouTube during the COVID-19 lockdown: implications on the risk of cannabis use disorders
}

\author{
Tianze Sun ${ }^{a, d}$, Carmen Lim ${ }^{a, b, c}$, Gary Chan ${ }^{a}$ and Janni Leunga,d
}

Publisher's Note: MDPI stays neutral with regard to jurisdictional claims in published maps and institutional affiliations.

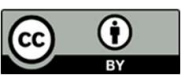

Copyright: ( 2020 by the authors. Submitted for possible open access publication under the terms and conditions of the Creative Commons Attribution (CC BY) license (http://creativecommons.org/licenses/by/4.0/).
aNational Center for Youth Substance Use Research, Faculty of Health and Behavioural Sciences, The University of Queensland, St Lucia QLD 4072, Australia

bQueensland Brain Institute, The University of Queensland, St Lucia QLD 4072, Australia 'Queensland Centre for Mental Health Research, The Park Centre for Mental Health, Wacol QLD 4076

dSchool of Psychology, The University of Queensland, St Lucia QLD 4072, Australia

* Correspondence::Tianze Sun tianze.sun@uq.net.au

\begin{abstract}
YouTube is a catalyst for influencing behaviour. During the pandemic, many found themselves turning to platforms like YouTube as a source of information and entertainment. This paper investigated the content of cannabis videos on YouTube during COVID-19. The impact of COVID-19 on cannabis use in the community was assessed by identifying 75 YouTube videos uploaded from March-May of 2020. We found that the contents of these videos display the impact of COVID-19 on cannabis use through four key reoccurring themes; (1) buying cannabis (45\%), (2) promotion of health claims (36\%), (3) the push for legalization (13\%) and (4) safety information (5\%). More vigorous monitoring and
\end{abstract}

tighter restrictions around social media is required to mitigate the risk of misinformation and normalisation, especially among those who are most vulnerable, such as individuals with cannabis use disorder.

Keywords: COVID19; cannabis; social media

\section{Introduction}

The outbreak of the Coronavirus Disease (COVID-19) has had a major impact on our mental health (Lima et al., 2020). Experiences of negative emotions such as loneliness, anxiety, stress and depression are common. Some may attempt to relieve them by using substances such as cannabis. Higher levels of cannabis use increase the risks of cannabis use disorders (CUD) (Leung et al., 2020). Therefore, there are concerns that COVID-19 may increase CUD by increasing the frequency or amount of use among people who use cannabis (Mota, 2020). Additionally, COVID-19 may even jeopardize the road to recovery for people with existing CUD.

With many of us stuck at home during this pandemic, some of us find ourselves turning to social media platforms like YouTube as a source of information and entertainment (Koeze and Popper, 2020). YouTube has seen an emergence of cannabis-related videos during COVID-19. The content of these videos may be a source of misinformation (Cuan-Baltazar et al., 2020) and an important means of influence during these challenging times. Thus, we aim to examine cannabis-related content on YouTube during COVID-19 in order to understand the messages being portrayed. This can provide an insight on the role of YouTube in influencing cannabis use behaviour, particularly for those who are most vulnerable, such as individuals at an increased risk of cannabis use during the lockdown. 


\section{Methods}

To explore the main themes, publicly available YouTube videos uploaded from March to May of 2020 were identified using the default filter parameter ("relevance") on YouTube. We used the phrase "cannabis covid-19" for our search term. Two reviewers independently watched and performed in vivo coding on 75 videos listed in Appendix 1 to identify recurring video themes. Ethical clearance was obtained from the Office of Research Ethics at the University of Queensland (exemption reference: 20200011080).

\section{Results}

We included a random sample of 75 YouTube videos in our study. Many of the videos assessed the current cannabis landscape during COVID-19 and were uploaded by news outlets. These videos were predominately from the United States and Canada. The identified video themes were: (1)'buying cannabis - content on consumer behaviour or the cannabis industry, (2) 'the push to legalization' - shifts and reforms in cannabis policy, (3) 'promotion of health claims' - messages that cannabis can prevent and treat COVID-19 and (4) 'safety information' - videos offering advice for safely consuming cannabis during COVID-19.

Approximately $45 \%$ of the videos (34/75) were on 'Buying Cannabis', 36\% (27/75) were on 'Promotion of health claims', 13\% (10/75) on 'Push for legalization' and 5\% (4/75) on 'Safety Information' (Figure 1). The total number of views across all 75 videos was 523,941. On average, these video were viewed between 24 - 70,453 times and received up to 4,404 likes. Although our sample contained a large number of videos on 'buying cannabis', videos on 'promotion of health claims' received a higher number of likes compared to videos from other themes.

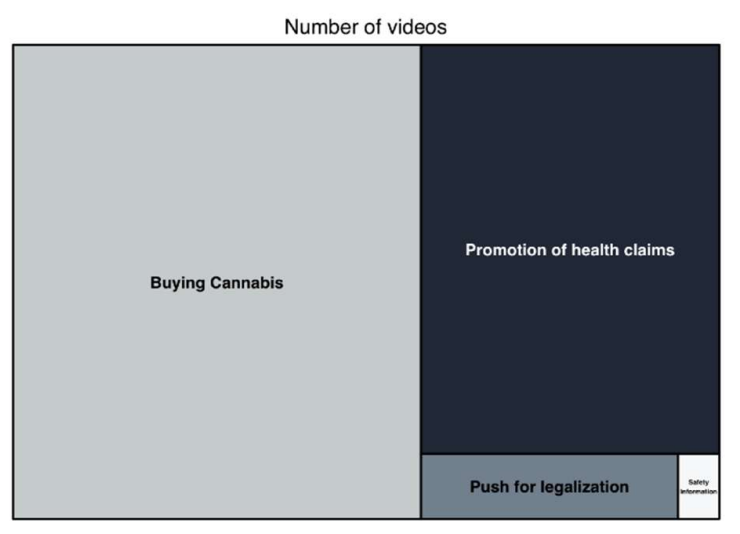

Number of likes

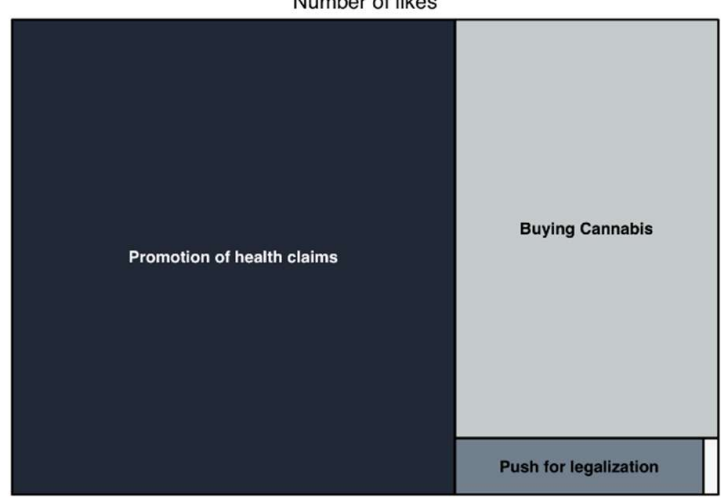

Number of views

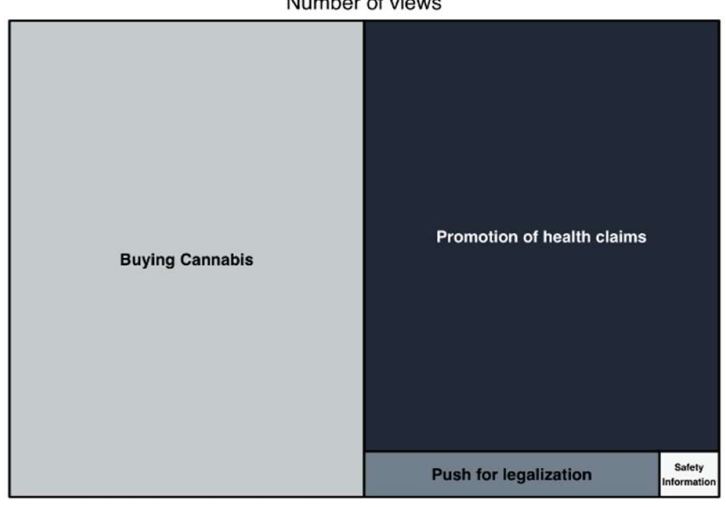

Number of dislikes

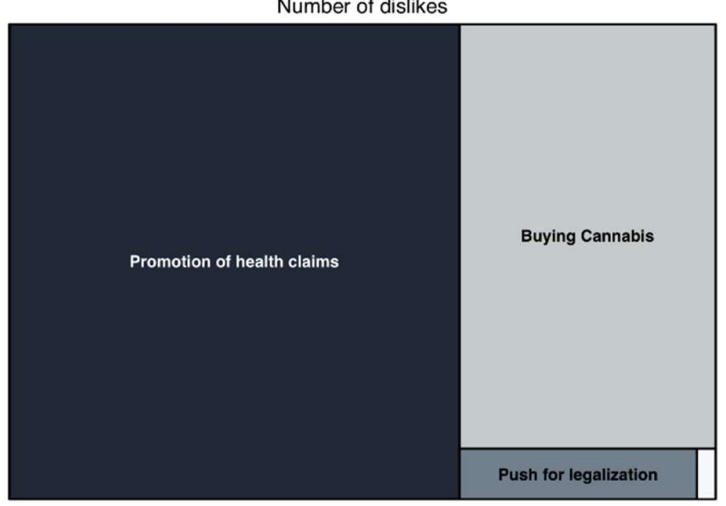


Figure 1. Treemap on number of videos, views, likes and dislikes for cannabis-related videos on YouTube by each video theme. Each rectangle has an area proportional to the amount of data it represents.

\subsection{Buying Cannabis}

As governments began to shut down parts of the economy, it became apparent from these videos that cannabis was one of the many items customers sought after. Footage of customers forming atypical long lines were captured by individuals and posted online. People were asked to stand six foot away as lines swerved around building blocks and stretched across car parks. There was an influx of videos on journalists reporting the spike in cannabis sales. Business owners expressed that their sales in cannabis increased exponentially before cannabis dispensaries were deemed as an essential service.

\subsection{The Push to Legalise Online Sales}

Legislators spoke out on how the pandemic has shifted the momentum away from cannabis legalization efforts. Calling out and reminding their audience for continuous support. For Colorado, the impact of COVID-19 has pushed legislators to legalise the online sale of recreational cannabis (Peipert, 2020). Some business owners expressed that they have advocated for these reforms before the pandemic, with hope that the changes can stay permanent.

But, when cannabis remains illegal at the federal level in the US, it can be expected that cannabis industries will be faced with a unique challenge. For example, The World Health Organization warned that cash could spread the novel coronavirus and advocated for the use of contactless payments (Pietsch, 2020). However, store managers in these videos acknowledged that this is unviable since banks and credit card companies are prohibited to work with the cannabis industry. Additionally, dispensary owners are unable to apply for a federal stimulus package or bank loans (Albergotti, 2020), forcing many to be self-reliant.

\subsection{Promotion of Health Claims}

The alleged benefits of cannabis claimed by cannabis advocates contributed largely to the content of these videos. Unscrupulous cannabis manufacturers and stores are taking advantage of consumer anxiety during the pandemic. Despite a lack of scientific evidence, some promoted medical benefits of their cannabidiol (CBD) products and claimed that it can assist with boosting immunity against and even treat COVID-19. A Canadian study on preventative effects of cannabis sativa on treating COVID-19 (Wang et al., 2020) is continuously cited in these YouTube videos. For example, in one video, a man reads the study's results and spends the latter half promoting the cannabis products. There were also videos representing and speaking to individuals who were diagnosed with COVID19. The patients would express how using cannabis has assisted with alleviating their symptoms of coronavirus (e.g., body pains). This may contribute to the current proliferation of unregulated and untested cannabis products in the marketplace (U.S. Food and Drug Administration, 2020).

\subsection{Safety Information}

Some independent YouTube influencers are providing safety information for cannabis administration in a way that would not contribute to the spread of the illness. They address the unsanitary dangers of "puff puff pass" when using joints and bongs in times of COVID-19 and provide tips to reduce harm. They urged that routinely cleaning pipes, bongs and other smoking devices is paramount during this time. Many also suggested administrating cannabis through edibles. Whilst others encouraged the complete prohibition from any type of smoking, linking smoking cannabis to negative health consequences that further exasperate the risk of contracting COVID-19.

\section{Concerns for the near future and need for action}


This research provided initial insights into how cannabis is portrayed on social media during the COVID-19 outbreak and how this information may impact the community. By tracking data from the latest uploads on YouTube, we provided information on a rapid snapshot of social media messages being spread in our society.

Businesses took advantage of the pandemic to increase their sales. This was evident from the large proportion of videos involving individuals spreading misinformation about the protective effects of cannabis for COVID-19 on YouTube (Ouellette et al., 2018). With the ease of purchasing cannabis as a recreational drug in the US, this misinformation may increase cannabis use for those with a higher susceptibility of CUD. For those who are more susceptible to CUD, social support is a critical protective factor. Unfortunately, with social isolation being a product of the pandemic, COVID-19 has increased the risk of CUD for individuals who may be more susceptible.

A minority of videos involved YouTubers encouraging their audiences to avoid the spread of the virus by promoting "safe" cannabis use behaviours, such as switching to edibles. As cannabis sales soared during the outbreak of panic buying behaviours, edibles were more likely to be sold than pre-rolled joints (Daily Chart, 2020) suggesting that consumers were aware of the unsanitary dangers of "puff puff pass". However, the shift towards administrating cannabis via edibles may be associated with a range of public health consequences, such as children unassumingly eating edibles or adults taking in more THC - the principle psychoactive contituent of cannabis, than intended (Leung et al., 2019).

YouTube's great influence, comes with even greater responsibility. Therefore, the millions of active users from across the globe that use YouTube as a tool to disseminate advice and guidance need to provide information that is accurate or risk their video not only demonetised, but removed. More vigorous monitoring and tighter restrictions around social media is required to mitigate the risk of problem cannabis use and CUD, especially among those who are most vulnerable.

Funding:This research did not receive any specific grant from funding agencies in the public, commercial, or not-for-profit sectors.

Declaration of Competing Interest:None.

[Insert Appendix 1 here.]

\section{References}

Albergotti, R., 2020. Weed is deemed 'essential' in California, but many pot businesses are on the brink of failure. Washington Post https://www.washingtonpost.com/business/2020/04/14/california-weed-industry-coronavirus (accessed June 4 2020)

Cuan-Baltazar, J. Y., Muñoz-Perez, M. J., Robledo-Vega, C., Pérez-Zepeda, M. F. \& Soto-Vega, E., 2020. Misinformation of COVID-19 on the internet: infodemiology study. JMIR Public Health Surveillance, 6 (2), e18444. https://doi.org/10.2196/18444

Daily Chart, 2020. Coronavirus lockdowns have caused Americans to stock up on pot. The Economist. https://www.economist.com/graphic-detail/2020/04/20/coronavirus-lockdowns-have-caused-americans-to-stock-up-on-pot (accessed June 4 2020).

Koeze, E. \& Popper, N., 2020. The Virus Changed the Way We Internet. The New York Times. https://www.nytimes.com/interactive/2020/04/07/technology/coronavirus-internet-use.html (accessed June 4 2020). 
Leung, J., Chiu, V., Chan, G. C. K., Stjepanović, D. \& Hall, W. D., 2019. What Have Been the Public Health Impacts of Cannabis Legalisation in the USA? A Review of Evidence on Adverse and Beneficial Effects. Current Addiction Reports, 6, 418-428. https://doi.org/10.1007/s40429-019-00291-X

Leung, J., Chiu, V., Chung, J. Y. C., Hall, W. D. \& Chan, G. C. K., 2020. How many more young Australians say that they would use cannabis if it was legal? Drug and Alcohol Review. [Accepted for publication]

Lima, C. K. T., Carvalho, P. M. D. M., Lima, I. A. A. S., Nunes, J. V. A. d. O., Saraiva, J. S. S., de Souza, R. I., da Silva, C. G. L. \& Neto, M. L. R., 2020. The emotional impact of Coronavirus 2019-nCoV (new Coronavirus disease). Psychiatry Research, 287, 112915. https://doi.org/10.1016/j.psychres.2020.112915

Mota, P., 2020. Avoiding a new epidemic during a pandemic: the importance of assessing the risk of substance use disorders in the COVID-19 era. Psychiatry Research, 290, 113142 https://doi.org/10.1016/j.psychres.2020.113142

Ouellette, L., Cearley, M., Judge, B., Riley, B. \& Jones, J., 2018. Cooking with cannabis: the rapid spread of (mis) information on YouTube. The American journal of emergency medicine, 36, 1300-1301. https://doi.org/10.1016/j.ajem.2017.10.060

Peipert, T., 2020. Coronavirus fuels marijuana industry's push for online sales, delivery in Colorado [Online]. The Colorado Sun. https://coloradosun.com/2020/04/13/online-marijuana-sales-delivery-colorado-coronavirus (accessed June 4 2020)

Pietsch, B., 2020. Cash could be spreading the coronavirus, warns the World Health Organisation. Business Insider Australia. https://www.businessinsider.com.au/cashcould-spread-coronavirus-warns-world-health-organization-2020-3? $\mathrm{r}=\mathrm{US} \& \mathrm{IR}=\mathrm{T}$ (accessed June 4 2020).

U.S. Food and Drug Administration, 2020. FDA Regulation of Cannabis and Cannabis-Derived Products, Including Cannabidiol (CBD). https://www.fda.gov/newsevents/public-health-focus/fda-regulation-cannabis-and-cannabis-derived-products-including-cannabidiol-cbd (accessed June 4 2020)

Wang, B., Kovalchuk, A., Li, D., Ilnytskyy, Y., Kovalchuk, I. \& Kovalchuk, O., 2020. In Search of Preventative Strategies: Novel Anti-Inflammatory High-CBD Cannabis Sativa Extracts Modulate ACE2 Expression in COVID-19 Gateway Tissues. MDPI AG. https://doi.org/10.20944/preprints202004.0315.v1 